Theresa Thunig, Kai Nagel

\title{
Towards a robust and wide-area traffic signal control for inner-city areas
}

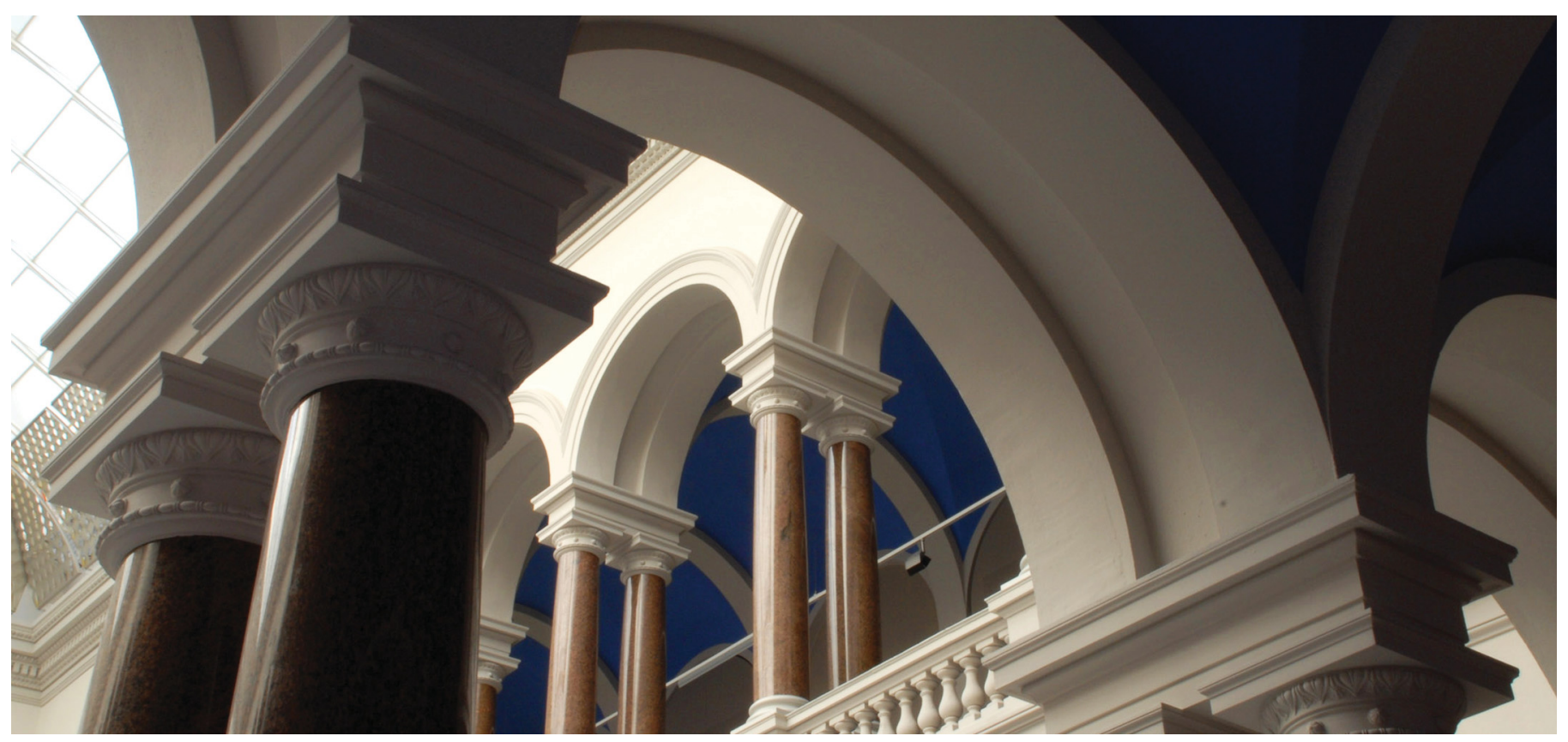

Thunig, T.; Nagel, K. (2017). Towards a robust and wide-area traffic signal control for inner-city areas. 2017 5th IEEE International Conference on Models and Technologies for Intelligent Transportation Systems (MT-ITS). https://doi.org/10.1109/MTITS.2017.8005622 


\title{
Towards a robust and wide-area traffic signal control for inner-city areas
}

\author{
Theresa Thunig and Kai Nagel \\ Transport Systems Planning and Transport Telematics \\ Technische Universität Berlin \\ Salzufer 17-19, 10587 Berlin \\ Email: thunig@vsp.tu-berlin.de
}

\begin{abstract}
This paper examines different signal approaches to control inner-city traffic and compares them with respect to two use cases - an illustrative gridlock scenario and a more complex real-world scenario. Based on the idea of back-pressure, it aims to develop an appropriate signal control method for inner-city traffic that reacts to current traffic and considers route choice, oversaturation, and spillback. For evaluation, the agent-based transport simulation MATSim is used. First positive results are presented. Difficulties that occur while applying the approach to the more complex scenario are analyzed. The outlook discusses suggestions to address these difficulties in the future.
\end{abstract}

\section{INTRODUCTION}

Today, more than half of the world population lives in cities, with increasing tendency. Intelligent transport control mechanisms are needed to coordinate the increasing city traffic. A widely-used control mechanism for inner-city traffic is signal control. Traffic signals influence travel time on routes and therefore influence user behavior, e.g. route choice, in the long term. A goal of signal optimization is to "push" users towards routes that reduce total travel time. Such optimization approaches already exist, but usually assume stationary demand pattern (e.g. [1]). In reality, however, traffic demand is not stationary. A network-wide optimization of signals for arbitrarily changing demand is challenging. There are approaches that address the problem from a more local perspective based on sensor data. Traffic-actuated signals adapt an underlying plan to current sensor data. They can handle changing demand and also unexpected events, but lack in network-wide optimization. Sensor-based signals usually do not influence users' route choice towards a system-optimal route distribution, but reduce travel time on already chosen routes. Another disadvantage is that adaptive signals usually cannot handle demand that exceeds capacities, i.e. oversaturation (e.g. [2]). It is wellknown that a route improvement attracts more users in reality (induced traffic). The number of travelers through a city, for instance, will increase by improving signal control inside the city. Inner-city signal control has to be able to deal with this phenomenon and keep traffic outside the city to prevent system break down (e.g. gridlock).

This paper aims to develop an appropriate signal control method for inner-city traffic based on the discussed criteria, that considers route choice, oversaturation, and spillback based on sensor-data. For evaluation, the agent-based transport simulation MATSim [3] is used.
In a first step, section III examines possibilities of different traffic control methods to prevent gridlock. A fixed-time and a local delay-minimizing traffic-actuated signal approach are compared to a signal control preventing congestion on downstream links (back-pressure). It is found that the approach based on the idea of back-pressure is the only one preventing gridlock. In a second step, the signal approaches are applied to a more complex real-world scenario including route choice in section IV. As expected, simple local delay-minimizing signals experience difficulties with oversaturation: Inner-city travel times increase, some agents switch to alternative bypass routes, but congestion in the inner city remains. As opposed to this, the back-pressure approach has the potential to keep congestion out of the city center without increasing innercity travel times. Difficulties that appear because of the more complex real-world scenario are analyzed in section IV-C. Solutions to extend the signal approach to capture these difficulties are discussed in section $\mathrm{V}$.

\section{PRELIMINARIES}

\section{A. Traffic signals and user reaction}

Traffic signal control can be classified into two groups: Traffic that follows signals, and signals that follow traffic. Both cases provide different possibilities of influence. The latter usually follows local optimization based on sensor data whereas the former is able to pursue network-wide (off-line) optimization, usually based on stationary traffic flows. The foundation of a traffic-follows-signals approach is that users learn from day to day and adapt their behavior to changes in signal control, e.g. by route, mode, or time choice. Networkwide effects of signal control changes can be considered, and individual optimization of users can be transformed towards system-wide optimization. On the other hand, signals that follow traffic usually work as standalone systems, which makes them applicable for large-scale scenarios. They optimize their actions based on local measurements and can, therefore, react to changes in current traffic. Potentially, this also improves the network-wide situation. This paper contributes to the understanding of this combination and the development of standalone signals that control traffic towards network-wide improvements. Thereby, it focuses on gridlock prevention and handling of oversaturation and spillback. 


\section{B. Back-pressure control in the literature}

The idea of back-pressure comes from communication systems, where network throughput is sought to be maximized by intelligent routing [4]. The central idea is to evaluate the pressure (e.g. density) on downstream links while deciding about traffic control on upstream links, e.g. by route guidance or traffic signal control. Thereby, it is distinct from common sensor-based signal control approaches that only evaluate queuing length at the junction itself.

With its evaluation of downstream traffic, it is a common approach for ramp metering, as vehicles on the highway are usually preferred over vehicles that try to enter the highway. If too many vehicles are held back, however, urban traffic can be influenced by spillbacks, see e.g. Taale et al. [5] who develop and analyze metering traffic lights that aim to hold vehicles back at different upstream (so-called buffer) locations to decrease flow on the on-ramp. Tackling the problem from a more local perspective, back-pressure approaches generally try to balance pressure on incoming and outgoing links of a junction. A common approach (e.g. [6], [7], [8], [9]) is to weight pressures (e.g. density or delay) at outgoing links e.g. by estimated or recorded turning rates, and to build on the difference between pressure at the incoming link and this weighted downstream pressure. This can be done for every incoming link of a junction, and can be used e.g. as a priority measure to arrange green splits of a signal plan, or to switch green phases adaptively. Additionally, some approaches weight each pressure difference by the maximum throughput (see [7], [8], [9]). Under the assumption of point queues (i.e. links with infinite storage capacity), a back-pressure-controlled junction is proven to maximize throughput and, if implemented at every junction, maximizes throughput of the whole network [4], [7]. Because queues are kept bounded, it also stabilizes the network at least for small unexpected events.

For the more realistic case of spatial queues that may fill up a link and lead to spillback effects on upstream links, the back-pressure approach does not necessarily maximize throughput anymore. Still, the idea of back-pressure gives useful insights for developing a decentralized control with network-wide effects in a more realistic and more complex environment. As back-pressure seams to be a good approach to prevent gridlock, it should be analyzed in combination with oversaturation and spillback. For the application of backpressure to signal control design, existing studies leave room for more flexible approaches. This paper contributes to this by providing a flexible back-pressure extension that can be combined e.g. with adaptive signals. Additionally, models used in previous studies differ in route selection and user reaction in general. Implementing the approach in another simulation environment may give new insights on the interaction of signal control and user reaction. Especially because back-pressure is commonly used for both, route choice and signal control, as e.g. in [9]. The transport simulation MATSim used in this paper (see section II-D) provides a back-pressure-independent route choice model.

\section{Back-pressure control in this study}

The signal control implemented for this study works as an extension of an existing signal approach. This means that it does neither contain a logic to build a signal plan nor to switch phases. Instead, it can be seen as an additional tool that controls saturation on downstream links. With this, a weighting of downstream pressure values as e.g. in [8], where pressures are squared to give more influence to higher values, is not necessary. The tool works as a hard bound: If the bound is exceeded, no more vehicles are allowed to enter the link, i.e. signals are switched to red. In contrast to other backpressure approaches, it therefore concentrates on the evaluation of saturation on downstream links and leaves the evaluation of upstream links to the underlying signal approach. In this paper, the approach is tested for fixed-signal plans as a basis. This combination turns out to be not ideal, as it has no possibility to switch phases when downstream sensors detect saturation. Instead, junction capacity remains unused which leads to problems when demand is increasing, see section IV-C). Still, it gives first insights and prepares for the combination with more complex signal controllers.

The implementation works as follows: The underlying signal plan is executed; to prevent congestion on downstream links that may lead to gridlock, sensors on downstream links report every second whether pressure is still below a specified threshold. If not, all signals controlling the inflow to this link are immediately switched to red. However, the underlying signal plan is still operating in the sense that active phases are not switched. It re-checks every second (until the end of the green phase defined in the base-case fixed-time plan) whether pressure on downstream links has decreased and active phases can again be switched to green. As an estimation of pressure, a combination of saturation and delay (as the difference to freespeed travel time) was used, similar to [8]. Note that common sensor-based signals (e.g. SYLVIA) only evaluate saturation numbers and not travel times. In this study, sensors report occupancy when free speed travel time cannot be reached anymore, or when at least $3 / 4$ of the storage capacity of the link is used, i.e. the spatial queue of vehicles on the link occupies at least 3/4 of the link length. As future green times on downstream junctions are not known and the approach aims to only rely on sensor data (vehicle counter at each beginning and end of a link), travel time estimations constitute a lower bound on the expected travel time of downstream links.

\section{The multi-agent transport simulation MATSim}

For evaluation the transport simulation MATSim [3] is used. MATSim is a tool designed for large-scale transport simulation scenarios, which makes the evaluation of network-wide effects possible. Its microscopic handling of agents that follow their daily plans supports sensor-based signal approaches. Flow dynamics are not explicitly modeled - links work as FiFo queues with flow capacity values that constitute the maximum outflow rate. As links possess a length and vehicles occupy physical space on the link, spatial queues can spill back and oversaturation can be modeled. Since recently, kinematic 


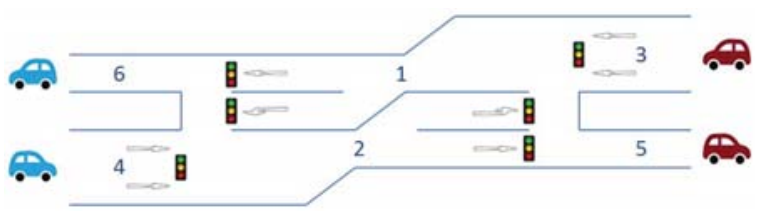

Fig. 1: Illustrative gridlock scenario

waves can be switched on [10], but that feature was not used for the present paper. MATSim works as an iterative learning dynamic, where in each iteration (e.g. each day) a share of agents is allowed to replan, to switch routes, modes, departure times etc. based on scores from the previous iteration (i.e. experiences from the previous day). As such, it is a tool suitable to analyze user reaction to signal control. Induced traffic is indirectly modeled in this study by the fact that largescale simulations are possible: Inside the study area (e.g. a city and its surroundings) vehicles may shift routes or switch modes when travel time through the city improves, which may induce additional traffic in the city.

\section{PREVENTING GRIDLOCK}

To motivate the development of a robust and wide-are traffic signal control for inner-city areas based on a back-pressure approach, this section presents a simple scenario, where both fixed-time and delay-minimizing signals lead to a gridlock, while a back-pressure approach is able to prevent it. Gridlock is defined as a state where vehicles block each other such that no vehicle can move anymore. A common example is a situation in a grid network, where all four streets around a block are occupied by queuing vehicles. When the first vehicles of each queue try to turn onto the street that leads around the block, no one can move at all. In reality, blocked intersections can also cause gridlocks. As MATSim does not microscopically model intersections, this section focuses on gridlock at links.

\section{A. Scenario}

The network shown in Fig. 1 is the smallest illustrative network where gridlock can occur. It consists of two symmetric bottleneck links in the middle - one for east-west direction (1), one for west-east direction (2) - and two incoming (3 and 4) and outgoing links (5 and 6) each. Every link has a length of $1 \mathrm{~km}$ and a free speed of $10 \mathrm{~m} / \mathrm{s}$. There are two symmetric commodities, one going from the western incoming link to the western outgoing link (blue) and the same for the eastern side (red). To reach its destination, the blue commodity has to enter the middle link and make a u-turn at the eastern side. The red commodity has make a u-turn at the western side. If all depicted signals show green all the time, a sufficiently large flow of both commodities unrestrictedly entering the middle link would result in a gridlock when the first vehicles of each commodity reach the u-turn because vehicles of the other commodity already block the other middle link.

To control inflow and to ideally prevent gridlock, the signals depicted in Fig. 1 can be operated. The next section explains three different signal approaches that are applied. To be able to depict and compare the demand levels for which the different signal approaches lead to gridlock, demand per commodity is increased stepwise during one simulated hour from one vehicle every four seconds to one vehicle every second, which constitutes the maximum throughput of the incoming links.

\section{B. Traffic signal control}

A fixed-time approach constitutes the base case. Fixed-time signals follow a predefined signal plan with a fixed cycle time and a specified order of phases each having a fixed amount of green time. The fixed-time plan used here, equally distributes green time to the two conflicting streams - the incoming link and the u-turn. The outgoing stream has green all the time. A cycle time of 60 seconds and intergreen times of one second are used. It is compared to a simple local delay-minimizing traffic-responsive signal based on the signal control algorithm SYLVIA [11]. SYLVIA consists of several modules that can be combined. A key feature is the traffic-actuated stage length control which is used here. A first version of SYLVIA has already been implemented in MATSim and been validated regarding its effect on exceptional events [12], [13]. In the implementation used here, SYLVIA is based on a fixed-time control, but initially shortens each green phase to 5 seconds; red times are not changed. When the fifth second of a green phase is reached, the phase is extended as long as vehicles want to leave the link. In this implementation, SYLVIA sticks to the cycle time of the underlying fixed-time signal plan. This has the effect that green phases coming first are privileged which leads to instabilities when demand increases. By using maximum extension times for all phases, e.g. based on the fixed-time phase length, and allowing for arbitrary cycle times, the local delay-minimizing signal falls back to the underlying fixed-time control when demand becomes too high. This stabilizes the approach (as green phases coming first are no longer privileged) and leads to better results, as shown in section IV-B. Note that flexible cycle times overwrite phase coordination between different junctions (as needed for green waves) if existing. The third signal control considered here is the implemented back-pressure approach discussed in section II-C. It is also based on the base-case fixed-time plans.

\section{Results}

Fig. 2 illustrates cumulated inflow (solid) and outflow (dashed) values of the middle links over time for the different signal control methods. An all-green approach is given as benchmark, which permanently shows green to all signals (green in Fig. 2). It results in a gridlock at second 2358 of the simulation (outflow is reduced to zero). The inflow slope shows that demand in this time period belongs to the second step (one vehicle every three seconds). Before gridlock, 945 vehicles have passed the network. The fixed-time approach (blue) restricts the inflow to the middle link to an average of one vehicle every two seconds (as it shows green half of the time for incoming vehicles). Since gridlock has occurred even earlier in the all-green case (for one vehicle every third 


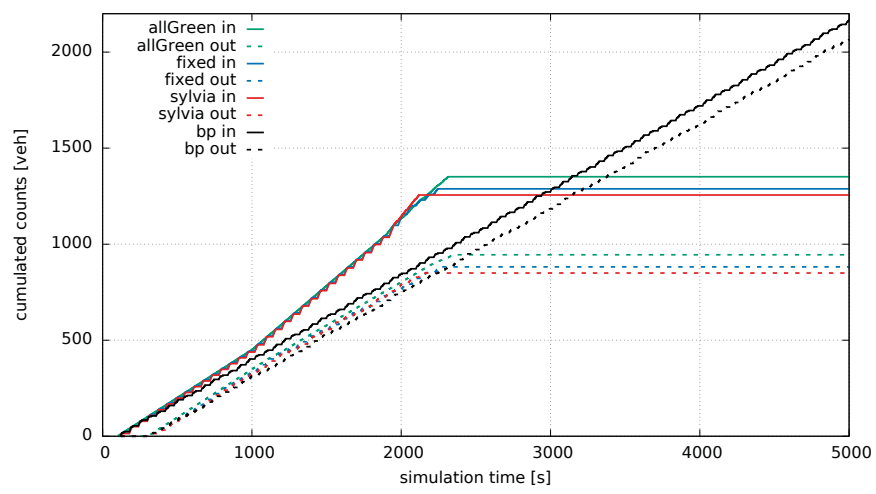

Fig. 2: Number of cumulated inflow and outflow of the middle links under different signal control approaches.

second), this specific fixed-time signal is not able to prevent gridlock. It even causes gridlock a bit earlier, as it clusters the vehicles in one-each-second platoons. SYLVIA (red) also promotes gridlock in this scenario, since it pushes as many vehicles as possible from the incoming link into the bottleneck, which simultaneously reduces green time for the u-turn. This causes the gridlock already at second 2140 with only 850 passed vehicles. The back-pressure approach (black) is able to prevent gridlock by limiting the number of vehicles in the bottleneck. It results in a constant inflow rate of approx. one vehicle every four seconds. Additional vehicles queue on the incoming links. Outflow stays slightly below the other approaches, but parallel to the inflow rate, which indicates stability. Note that there are also fixed-time plans that can prevent gridlock by restricting the inflow to one vehicle every four seconds, e.g. by giving more green time to the u-turn. The aim here is to show that one does not have to know about exact gridlock properties to design a suitable fixed-time plan. One can simply let sensors check the current traffic situation and decide for a control automatically.

\section{A MORE COMPLEX USE CASE}

Based on the promising results from the illustrative scenario, the back-pressure approach is now applied to a more complex, real-world scenario, where agents can react to signal control changes by choosing a new route ("re-route").

\section{A. Scenario}

The scenario represents the city of Cottbus, Germany and its surroundings as shown in Fig. 3. It is based on the scenario of Grether [12], where demand was generated from commuter statistics. For this study, only the morning-peak traffic consisting of 33,302 home-work trips is analyzed. The scenario is simulated for 100 iterations. In each iteration, $10 \%$ of the agents are allowed to re-route. Optimized fixedtime signal plans exist for the 22 intersections in the inner city depicted in Fig. 3 (see [12], [14]). This serves as base case. They are compared with both signal approaches from section III-B. Flow capacity is artificially decreased to analyze

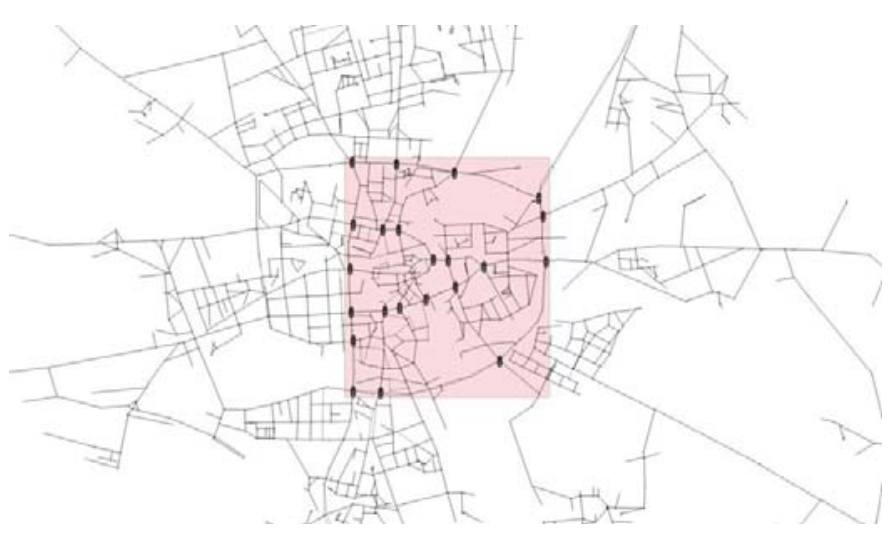

Fig. 3: Cut-out of the used network of Cottbus and its surroundings. Signals are simulated only in the inner city area.

the signals' reaction to oversaturation. As the simple delayminimizing SYLVIA approach presented in section III-B is unable to cope with higher demands, it is compared to the stabilizing variant also discussed in section III-B that falls back to its underlying fixed-time plan when demand becomes too high. An all-green signal approach serves as a benchmark.

\section{B. Results}

As Fig. 4a shows, total travel times for all signal approaches increase for increasing traffic (i.e. decreasing flow capacity factors). Not contained in the figure are the numbers of stuck agents, which indicate gridlock situations. For a flow capacity factor of 0.3 , i.e. a reduced flow capacity to $30 \%$ of the actual capacity, all presented signal approaches result in gridlock. For $40 \%$ most of them result in gridlock, at least congestion is uncontrollably high, also for the all-green benchmark (solid green in Fig. 4), which constitutes a lower bound to the total travel time for all cases. The highest density value for which meaningful results can be compared corresponds to the flow capacity factor of 0.5 : Saturation seems to be manageable at least for suitable signal control approaches.

An important result is that the simple local delayminimizing signal control based on SYLVIA (dotted black) gives unstable and high travel times for increasing density. This is due to an unfair processing of waiting queues based on the order of phases in the base plan (as mentioned in section III-B). Travel times for the inner-city area (see Fig. 4b) clarify this instability. The stabilized variant (dot-dot-dashed violet) that falls back to the underlying fixed-time plan for high demand (by using flexible cycle and maximal extension times) results in much better travel times, even lower than for the fixed-time control (dot-dashed red).

\section{Discussion}

The intuitive combination of the implemented back-pressure approach and the optimized fixed-time control (short-dashed blue) does not produce good results. Even for low density values, it results in an average trip travel time increase of almost 3 minutes. Interestingly, it produces lower travel times in the inner city (see Fig. 4b), which is due to a reduced number of 


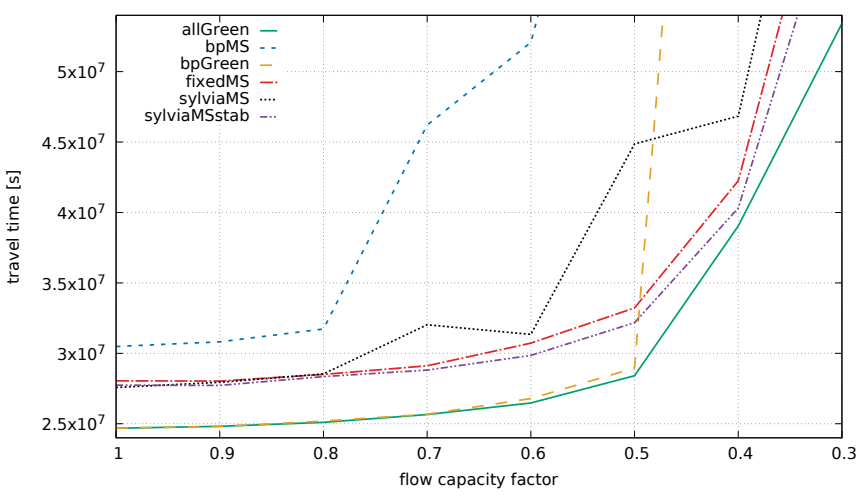

(a) full network

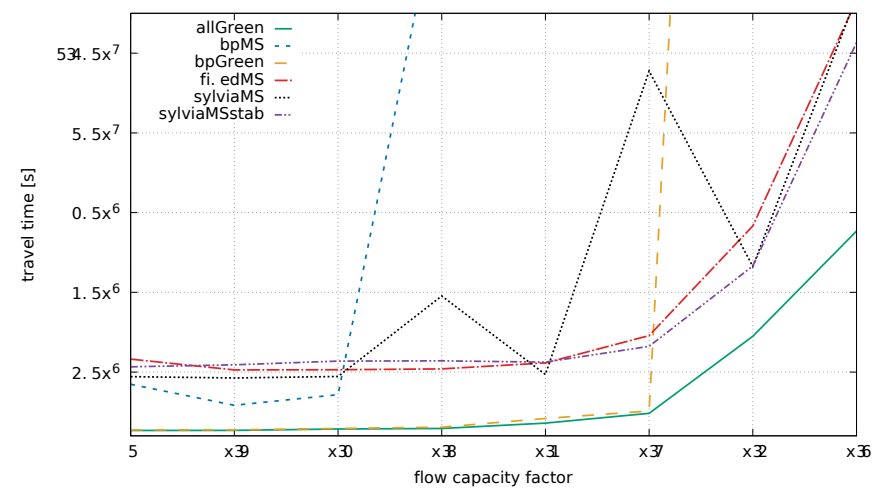

(b) inner-city network (colored bounding box in Fig. 3)

Fig. 4: Total travel times in the Cottbus scenario with different flow capacity values and signal approaches as described in section IV-B and IV-C.

trips (to around 60\%) through the inner city. This demonstrates the potential of the approach to keep vehicles outside of the city center without increasing inner-city travel times. On the other hand, there are difficulties with this application of the back-pressure approach in a complex scenario. Observing the reactions of the signals in a visualization of the simulation detects the following difficulties:

A) The approach in its simple implementation presented in section III-B cannot handle mixed lanes or signal groups, i.e. a signal that controls different turning directions at once. As soon as one of the downstream links detects congestion, all turning directions are switched to red irrespective of turning purposes of agents upstream of the signal. This even gets more complicated when opposing directions are grouped in one signal group. In both cases it promotes gridlock when only one outgoing link of the junction is congested.

B) When signals are switched to red because of downstream congestion, the control approach does not contain a logic that switches to the next phase. Instead, the junction is completely blocked in this seconds and capacity remains unused. This increases congestion when demand is high.

C) The sensors only check the first downstream link of each turning direction. Congestion on links further downstream can not be detected until spillback reaches the first downstream link. This leads to two issues: i) The lower the share of signalized intersections, the lower the impact of the approach, and ii) the shorter the downstream link, the smaller the influence and the lower the stability of each back-pressure signal.

D) In scenarios where no gridlock can occur, the implemented back-pressure approach is clearly not able to improve the situation. Indeed, it has the potential to worsen it as it artificially extends spillback and blocks agents that would not be influenced by bottleneck congestion otherwise. This can be useful to trigger agents to use alternatives, e.g. bypasses around the city, but does not help when no (good) alternatives exist.

These issues do not exist in the illustrative scenario from section III. Not even unused capacity (issue B) is a problem, as both signal phases of each junction lead to the same link - when it is occupied the whole junction has to show red anyway. This underlines the general importance of applying such theoretical approaches to more complex scenarios.

For the back-pressure approach based on fixed-time plans, all aforementioned difficulties come into effect simultaneously. To be able to analyze their effects separately, a simplified (clearly unrealistic) use case was created: According to issue A all signal groups were separated and separate lanes for all turns were created. To prevent issue $\mathrm{B}$, the all-green benchmark was used as the base plan. This makes the approach incomparable with the fixed-time and all other controls that respect conflicting streams and intergreen times; vehicles from conflicting streams can pass junctions at the same time in the simulation. To prevent difficulty $\mathrm{C}$, all junctions inside the inner city (the colored area in Fig. 3) were signalized and equipped with a back-pressure controller. The resulting signal approach (back-pressure at all inner-city junctions based on all-green signal plans with separated lanes for all turning directions) is included in Fig. 4 (long-dashed orange). It produces travel times similar to the all-green benchmark, i.e. it at least does not worsen the situation until a saturation volume that corresponds to a reduction of the flow capacity to $50 \%$. For a reduction to $40 \%$, it abruptly leads to gridlock caused by the extended spillbacks described in issue D.

Summarizing, the back-pressure logic itself has the potential to keep traffic outside the inner city without increasing innercity travel times. It can also handle non-gridlock situations as good as conventional approaches as long as demand does not exceeds a manageable level.

\section{OUTLOOK}

To reliably realize the potential of back-pressure control, one has to develop solutions for all difficulties presented above and extend the back-pressure implementation accordingly. 
Mixed lanes (issue A) are frequently found at small junctions. Signal control has to be able to deal with them. One idea that will be tested is to decide based on the direction of the first vehicles in the queue. In the simulation, one can easily get this information and only switch to red when downstream links of first vehicles (or the majority of next vehicles) is occupied. Nowadays, this does not seem to be overly unrealistic as autonomous vehicles and car-to-infrastructure communication is feasible. Another much simpler idea is to use information about previous or expected turning rates and weight the downstream occupancy according to these averages, as it is done in common back-pressure approaches (e.g. [7], [8], [9]).

To solve problem $\mathrm{B}$, the back-pressure logic will be combined with signal approaches that switch phases based on sensor data (as the SYLVIA approach presented here) or even decide for following signal phases on the fly (as the approach by Lämmer [2] which is currently being implemented in MATSim). This coupling of up- and downstream sensors aims to combine the advantages of both approaches: Minimizing delay of unexpected traffic and being able to deal with oversaturation and prevent gridlock.

Signalizing every junction, as done in the descriptive approach in section IV-C, and as it is assumed for most backpressure approaches (see [6], [7], [8], [9]), is not applicable in reality. Therefore, one has to come up with other ideas how to solve problem $\mathrm{C}$ effectively. One attempt is to determine a tree of downstream links for every outgoing link of a signalized junction. The tree contains all possible paths starting from the first downstream link and ending at signalized junctions. Sensor data of all tree links could be combined to weight overall downstream congestion according to average route usages or destinations of single vehicles similar to the solution presented above for the mixed lanes problem. Another similar, but lighter approach is to bundle the pressure of next downstream links in every link (e.g. weighted as discussed above via knowledge about route distributions). Sensors on downstream links could then report the pressure of the link itself together with the bundled and less weighted pressure of next downstream links consisting again of their downstream link pressures and so forth. Both approaches require more and wider communication between signals and sensors, but still seem realistic.

To test the proposed extensions of the back-pressure approach in the future, one has to find a scenario where users have more alternatives and overall improvement potential is higher (see issue D). Staying with the Cottbus scenario, one could add an alternative transport mode that does not interact with individual traffic and, thus, constitutes a stable alternative. With that, one would also be able to analyze scenarios with higher saturation since it increases the comparability of failing approaches. Additionally, the combined calculation of pressure based on incoming and outgoing links, as other back-pressure approaches like [6], [7], [8], [9] suggest, probably stabilizes the extension of spillback effects that block other agents when no alternatives exist. A combination with intelligent adaptive signals would probably have a similar effect as adaptive signals usually try to bound upstream queues.

\section{CONCLUSION}

This paper gives clear indication that the idea of backpressure is a suitable approach towards a robust and wide-area traffic signal control for inner-city areas. It is able to prevent gridlock for unexpected traffic demands, it can keep traffic outside of a city without increasing inner-city travel times (i.e. is able to deal with induced traffic), and it has the potential to handle non-gridlock situations as good as conventional signal approaches. Important difficulties that have to be solved for making the approach applicable to more complex use cases have been discussed. Further steps to solve them have been suggested. Finally, the paper states that the applied Cottbus scenario does not provide a suitable use case to test the backpressure approach regarding its effect to route choice.

The results for fixed-time and local delay-minimizing signal approaches compared for different density values also give new insights. The comparison of the SYLVIA approach with and without stabilization rule shows that local delayminimization is not good per se.

\section{REFERENCES}

11] E. Köhler and M. Strehler, "Traffic signal optimization using cyclically expanded networks," Networks, vol. 65, no. 3, pp. 244-261, 2015.

[2] S. Lämmer, "Reglerentwurf zur dezentralen Online-Steuerung von Lichtsignalanlagen in Straßennetzwerken," Ph.D. dissertation, Dresden Institute of Technology, 2007.

3] A. Horni, K. Nagel, and K. W. Axhausen, The Multi-Agent Transport Simulation MATSim, A. Horni, K. Nagel, and K. W. Axhausen, Eds. Ubiquity, London, 2016. [Online]. Available: http://matsim.org/the-book

[4] L. Tassiulas and A. Ephremides, "Stability properties of constrained queueing systems and scheduling policies for maximum throughput in multihop radio networks," IEEE Transactions on Automatic Control, vol. 37, pp. 1936-1948, 1992.

[5] H. Taale, S. Hoogendoorn, and P. Legius, "Metering with traffic signal control - development and evaluation of an algorithm," Transportation Research Procedia, vol. 8, pp. 204-214, 2015.

[6] P. Varaiya, "Max pressure control of a network of signalized intersections," Transportation Research Part C: Emerging Technologies, vol. 36, pp. 177-195, 2013.

[7] T. Wongpiromsarn, T. Uthaicharoenpong, Y. Wang, E. Frazzoli, and D. Wang, "Distributed traffic signal control for maximum network throughput," CoRR, 2012.

[8] H. Taale, J. van Kampen, and S. Hoogendoorn, "Integrated signal control and route guidance based on back-pressure principles," Transportation Research Procedia, vol. 10, pp. 226-235, 2015.

[9] A. Zaidi, B. Kulcsár, and H. Wymeersch, "Decentralized traffic signal control with fixed and adaptive routing of vehicles in urban road networks," IEEE transactions on intelligent transportation systems, vol. 17, no. 8, pp. 2134-2143, 2016.

[10] A. Agarwal, G. Lämmel, and K. Nagel, "Modelling of backward travelling holes in mixed traffic conditions," in Traffic and Granular Flow '15, V. L. Knoop and W. Daamen, Eds. Delft, NL: Springer International Publishing, 2016, pp. 419-426.

[11] J. Krimmling, "Sytem SYLVIA; Verkehrstechnische Beschreibung der Steuerverfahren," Dec. 1995, monograph type: technical report; department: ANL-SV.

[12] D. S. Grether, "Extension of a multi-agent transport simulation for traffic signal control and air transport systems," Ph.D. dissertation, TU Berlin, Berlin, 2014

[13] D. Grether, J. Bischoff, and K. Nagel, "Traffic-actuated signal control: Simulation of the user benefits in a big event real-world scenario," in 2nd International Conference on Models and Technologies for ITS, Leuven, Belgium, 2011, also VSP WP 11-12, see http://www.vsp.tuberlin.de/publications.

[14] M. Strehler, "Signalized flows - optimizing traffic signals and guideposts and related network flow problems," Ph.D. dissertation, BTU Cottbus, 2012. 